\title{
ESTIMATES OF UNKNOWN TRANSFORMATION PARAMETERS IN TERRESTRIAL MEASUREMENTS: ONE SIMULATED PROBLEM
}

\author{
Nedvědová Marie $^{(a)}$, Marek Jaroslav ${ }^{(b)}$, Chmelař Pavel $^{(\mathrm{c})}$ \\ (a),(b),(c) University of Pardubice, Faculty of Electrical Engineering and Informatics, Studentská 95, 53210 Pardubice, \\ Czech republic \\ ${ }^{\text {(a) }}$ marie.nedvedova@student.upce.cz, ${ }^{\text {(b) }}$ jaroslav.marek@upce.cz, ${ }^{(c)}$ st19544@,student.upce.cz
}

\begin{abstract}
In connection with the expansion of 3D scanners, 3D object modeling has become highly studied in recent years. Many methods are currently available to solve the registration problem, whereby unknown transformation parameters need to be estimated when targeting a 3D object in multiple scans from different locations. Two different problems are encountered in the practice of targeting $3 \mathrm{D}$ objects in geodesy or construction. In the first variant, the measurement of the coordinates of the points of the $3 \mathrm{D}$ object is realized in several scans on tens of points marked with targets on a reflective surface. In the second variant, measurements of the coordinates of "clouds of hundreds or thousands of points" are available in several scans from different coordinate systems. In clouds it is necessary to find matching pairs of points, called identical points, based on their color match. In both versions, the coordinates of identical points from different coordinate systems must be recalculated to the selected coordinate system during data fusion. The problem leads to finding unknown shift and rotation transformation parameters. The aim of this article is to simulate the measurement of identical points in multiple scans. We will create a test task that can be used to test the methods proposed to solve the registration problem.
\end{abstract}

Keywords: registration problem, 3D range scanning, transformation of coordinates, point clouds

\section{INTRODUCTION}

The $3 \mathrm{D}$ range scans fusion is called registration. If the localization in a space or user's measurements are precise, the registration could be done directly by individual measurement connection into one group. However, due to inaccuracy of measurement sensors and the erroneous self-localization, the registration has to be considered.

In recent years, many methods have been developed to solve the registration problem that occurs in $3 \mathrm{D}$ scanning of objects. 3D cameras are sources of a large set of measurement points. When needed to recognize a $3 \mathrm{D}$ model of an object from the point clouds, an efficient method for identifying identical points is required. Obtained identical points are measured in different coordinate systems and it is necessary to find unbiased estimates of these transformation parameters.

The most commonly used algorithms are: ICP Algorithm (He, Liang, Yang, Li, and He 2017), Normal distribution transform (Magnusson 2013), Feature based registration (Nüchter 2009), Iterative dual correspondences (Lu and Milios 1997), Probabilistic iterative correspondence method (Montesano, Minguez, and Montano 2005), Quadratic patches (Mitra, Gelfand, Pottmann, and Guibas 2004), Likelihood-field matching (Burguera, Gonzalez, and Oliver 2008), Conditional random fields (Bataineh, Bahillo, Díez, Onieva, and Bataineh 2016), PointReg (Olsen, Johnstone, Kuester, Driscoll, and Ashford 2011). These method ensembles exhibit a lot of interesting properties, and required accuracy of estimation is widely met. Helmert transformation plays a key role, cf. (Amiri-Simkooei 2018). Three dimensional (3D) coordinate transformations are generally given by three origin shifts, three axes rotations, three scale changes and three skew parameters.

Unfortunately, in literature there exists no dataset with a simple testing problem with known solution of such a problem. Therefore, we will try to prepare such a test problem.

In this paper, the ICP algorithm will be presented in a very general manner without any assumptions of the point clouds feature to be assigned. A semi-automatic procedure for identic point segmentation, outlier elimination and transformation parameters estimation in point clouds will be explored on our testing problem.

\subsection{Basic ideas of ICP algorithm}

During the last years researchers used ICP very often, see (He, Liang, Yang, Li, and He 2017). The first reason is its easy feasibility. The second reason is almost no limits on point cloud size.

The algorithm calculates the optimal rotation and translation for the model to minimize the distances between the corresponding points.

In the first step, the algorithm tries to find matching pairs of points from both clouds.

In the second step, it updates the rotation matrix and the shift vector based on the initial point assignment. 
Then, according to the rotation matrix and the shift vector, it transforms a point cloud.

Given two independently acquired sets of 3D points from position $P_{1}$ and $P_{2}$, we want to find the transformation $(\boldsymbol{R}, \boldsymbol{t})$ consisting of a rotation matrix $\boldsymbol{R}$ and a translation vector $\boldsymbol{t}$ which minimizes the following cost function

$$
E(\boldsymbol{R}, \boldsymbol{t})=\sum_{i=1}^{N_{m}} \sum_{j=1}^{N_{d}} w_{i, j}\left\|\widehat{m}_{i}-\left(\boldsymbol{R} \widehat{d}_{j}+\boldsymbol{t}\right)\right\|^{2} .
$$

$w_{i, j}$ is assigned 1 if the $\mathrm{i}$-th point of $\widehat{m}_{i}$ describes the same point in space as the $\mathrm{j}$-th point of $\hat{d}$. Otherwise $w_{i, j}$ is 0 . Two things have to be calculated: First, the corresponding points, and second, the transformation $(\boldsymbol{R}, \boldsymbol{t})$ that minimizes $E(\boldsymbol{R}, \boldsymbol{t})$ on the base of the corresponding points. The ICP algorithm calculates iteratively the point correspondences. In each iteration step, the algorithm selects the closest points as correspondences and calculates the transformation $(\boldsymbol{R}, \boldsymbol{t})$ for minimizing equation $E(\boldsymbol{R}, \boldsymbol{t})$.

Indeed, on one hand, the quality of results is affected essentially by the camera accuracy. On the other hand, the number of correctly identified points in different scans is important.

Therefore, there are still many interesting open questions.

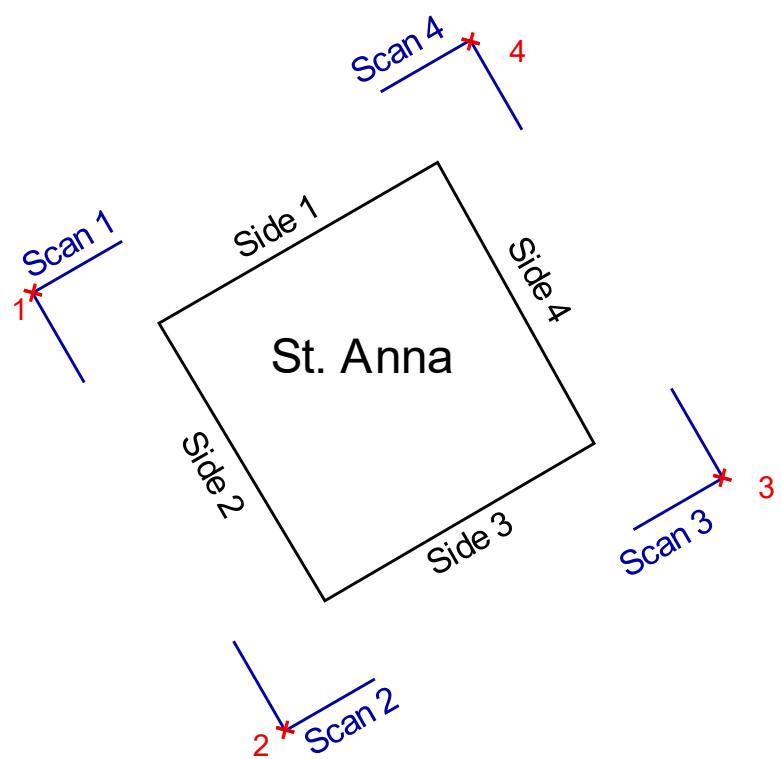

Figure 1: Chapel's plan and four coordinate systems

\section{ONE SIMULATED PROBLEM}

In the following subchapters we will present one simulated problem, the solution of which appears in Chapter 3.

We base our example on the 3D description of the Chapel of Saint Anna in Pardubice.

Consider that the actual geometric shape of the chapel's plan is an equilateral trapezoid. Next, let's work with measurements in four coordinate systems. See Fig. 1.

Next, we will prepare $\mathrm{X}, \mathrm{Y}, \mathrm{Z}$, and HSV color simulations of point clouds in 4 scans that will contain identical and non-identical points.
However, collecting of theoretical true values and noisy data are our interest.

Studies of covariance matrices of HSV are well suited for the investigation of color transformation of the same point between scans.

\subsection{The first step: coordinate simulation}

The similar numerical example with two scans is given in (Marek, Rak (2)015) that is focused on simulating only $3 \mathrm{D}$ coordinates in two scans.

Let us denote the northwest wall as side 1, the southwest wall as side 2, the southeast wall as side 3 and the northeast wall as side 4. See Fig. 1. We simulate point cloud measurements in 4 scans (two sides 1-2, 2-3, 3-4, 4-1 are scanned in each scan).

In this task, we simulate the positions of several thousand points in clouds for these scans.

We have the model given by

$$
\boldsymbol{Y}=\left[\begin{array}{c}
\boldsymbol{Y}_{1}^{I} \\
\boldsymbol{Y}_{2}^{I} \\
\boldsymbol{Y}_{2}^{I I} \\
\boldsymbol{Y}_{3}^{I I} \\
\boldsymbol{Y}_{3}^{I I I} \\
\boldsymbol{Y}_{4}^{I I I} \\
\boldsymbol{Y}_{4}^{I V} \\
\boldsymbol{Y}_{1}^{I V}
\end{array}\right]=\left[\begin{array}{c}
\boldsymbol{a}_{1}^{I} \\
\boldsymbol{a}_{2}^{I} \\
\boldsymbol{a}_{2}^{I I} \\
\boldsymbol{a}_{3}^{I I} \\
\boldsymbol{a}_{3}^{I I I} \\
\boldsymbol{a}_{4}^{I I I} \\
\boldsymbol{a}_{4}^{I V} \\
\boldsymbol{a}_{1}^{I V}
\end{array}\right]+\varepsilon
$$

$$
\boldsymbol{\varepsilon} \sim N(\mathbf{0}, \Sigma), \quad \Sigma=\left(\begin{array}{cccc}
\Sigma_{1} & 0 & 0 & 0 \\
0 & \Sigma_{2} & 0 & 0 \\
0 & 0 & \Sigma_{3} & 0 \\
0 & 0 & 0 & \Sigma_{4}
\end{array}\right)
$$

Notation of model $\boldsymbol{Y}=\boldsymbol{a}+\boldsymbol{\varepsilon} \sim N[\mathbf{0}+\boldsymbol{a}, \boldsymbol{\Sigma}]$ means that observation vector $\mathbf{Y}$ (with elements $\boldsymbol{Y}_{1}^{I}$ and $\boldsymbol{Y}_{1}^{I V}$ ) has (symbol $\sim$ ) multinomial normal distribution with mean value $\left(\boldsymbol{a}_{1}^{I}, \ldots, \boldsymbol{a}_{1}^{I V}\right)$ and with covariance matrix $\boldsymbol{\Sigma}$.

3ni-dimensional vector $\boldsymbol{a}_{1}^{I}$ is the vector of true coordinates $n_{i}$ points on $\mathrm{i}$-th side of object in a coordinate system of i-th device position. Analogous $\boldsymbol{a}_{1}^{I}$ is $3 n_{i+1^{-}}$ dimensional vector of $n_{i+1}$ points on $(i+1)$-th side of an object in a coordinate system of $\mathrm{i}$-th device position.

From layout of measurement we can obtain constraint function

$$
\boldsymbol{g}=\left[\begin{array}{l}
\boldsymbol{g}_{2}^{I I}\left(\gamma_{2}, \boldsymbol{T}_{2}\right) \\
\boldsymbol{g}_{3}^{I I}\left(\gamma_{2}, \boldsymbol{T}_{2}\right) \\
\boldsymbol{g}_{3}^{I I I}\left(\gamma_{3}, \boldsymbol{T}_{3}\right) \\
\boldsymbol{g}_{4}^{I I I}\left(\gamma_{3}, \boldsymbol{T}_{3}\right) \\
\boldsymbol{g}_{4}^{I V}\left(\gamma_{4}, \boldsymbol{T}_{3}\right) \\
\boldsymbol{g}_{1}^{I V}\left(\gamma_{4}, \boldsymbol{T}_{3}\right)
\end{array}\right]=\left[\begin{array}{l}
\boldsymbol{a}_{2}^{I I}-\gamma_{2}-\boldsymbol{T}_{2} \boldsymbol{a}_{2}^{I} \\
\boldsymbol{a}_{3}^{I I}-\gamma_{2}-\boldsymbol{T}_{2} \boldsymbol{a}_{3}^{I} \\
\boldsymbol{a}_{3}^{I I I}-\gamma_{3}-\boldsymbol{T}_{3} \boldsymbol{a}_{3}^{I} \\
\boldsymbol{a}_{4}^{I I I}-\gamma_{3}-\boldsymbol{T}_{3} \boldsymbol{a}_{4}^{I} \\
\boldsymbol{a}_{4}^{I V}-\gamma_{4}-\boldsymbol{T}_{4} \boldsymbol{a}_{4}^{I} \\
\boldsymbol{a}_{1}^{I V}-\gamma_{4}-\boldsymbol{T}_{4} \boldsymbol{a}_{1}^{I}
\end{array}\right]=0
$$

Notation of model $\boldsymbol{Y}=\boldsymbol{a}+\boldsymbol{\varepsilon} \sim N[\mathbf{0}+\boldsymbol{a}, \boldsymbol{\Sigma}]$ means that observation vector $\mathbf{Y}$ (with elements $\boldsymbol{Y}_{1}^{I}$ and $\boldsymbol{Y}_{1}^{I V}$ ) has (symbol $\sim$ ) multinomial normal distribution with mean value $\left(\boldsymbol{a}_{1}^{I}, \ldots, \boldsymbol{a}_{1}^{I V}\right)$ and with covariance matrix $\boldsymbol{\Sigma}$. 


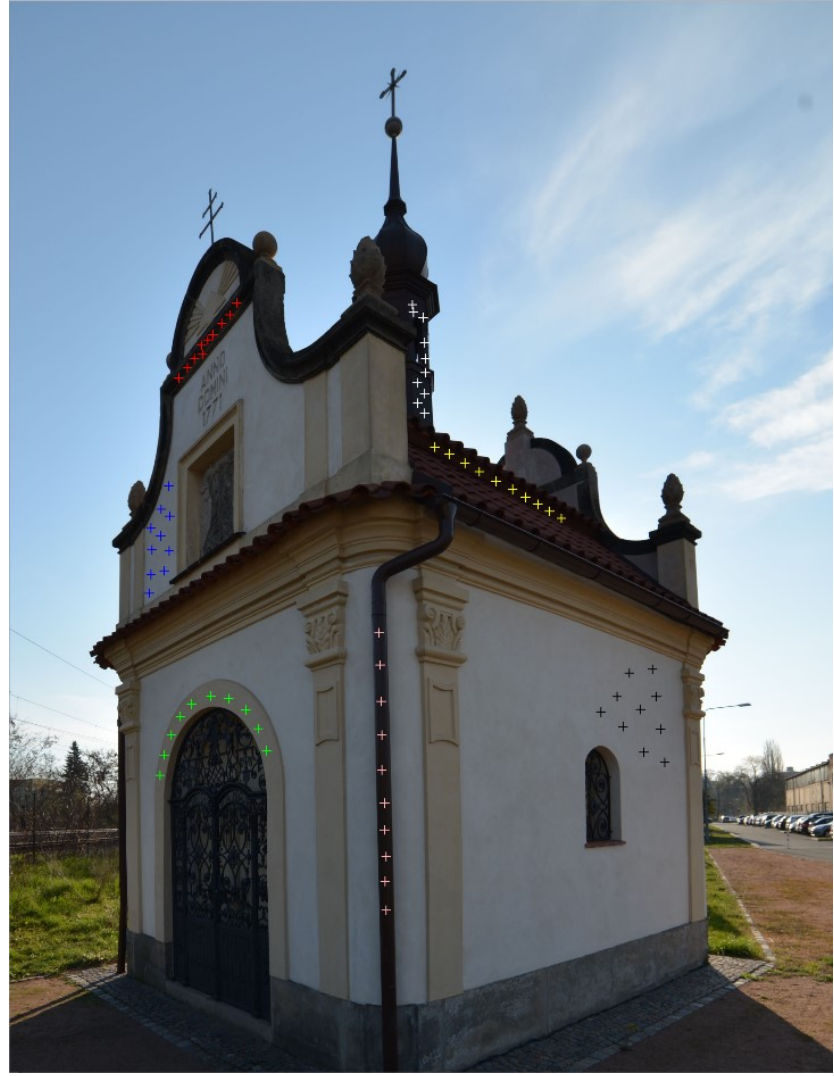

a) $1^{\text {st }}$ and $2^{\text {nd }}$ sides ( $\left.\operatorname{scan} 1\right)$

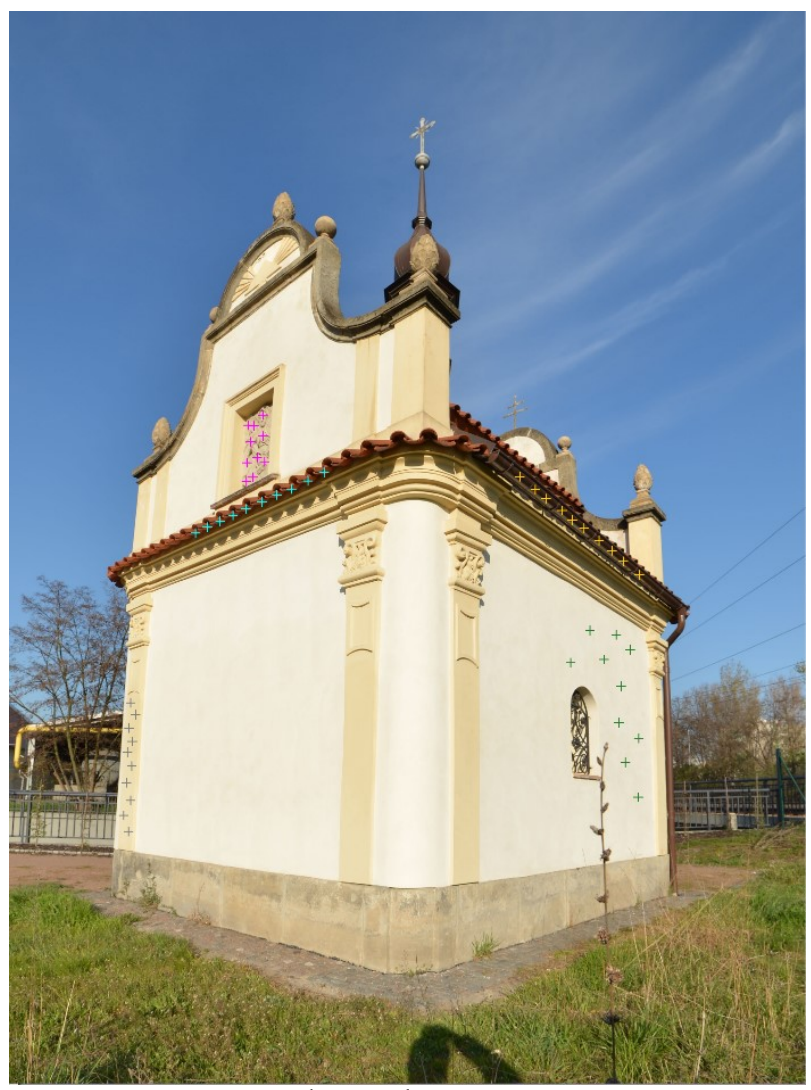

c) $3^{\text {rd }}$ and $4^{\text {th }}$ sides $(\operatorname{scan} 3)$

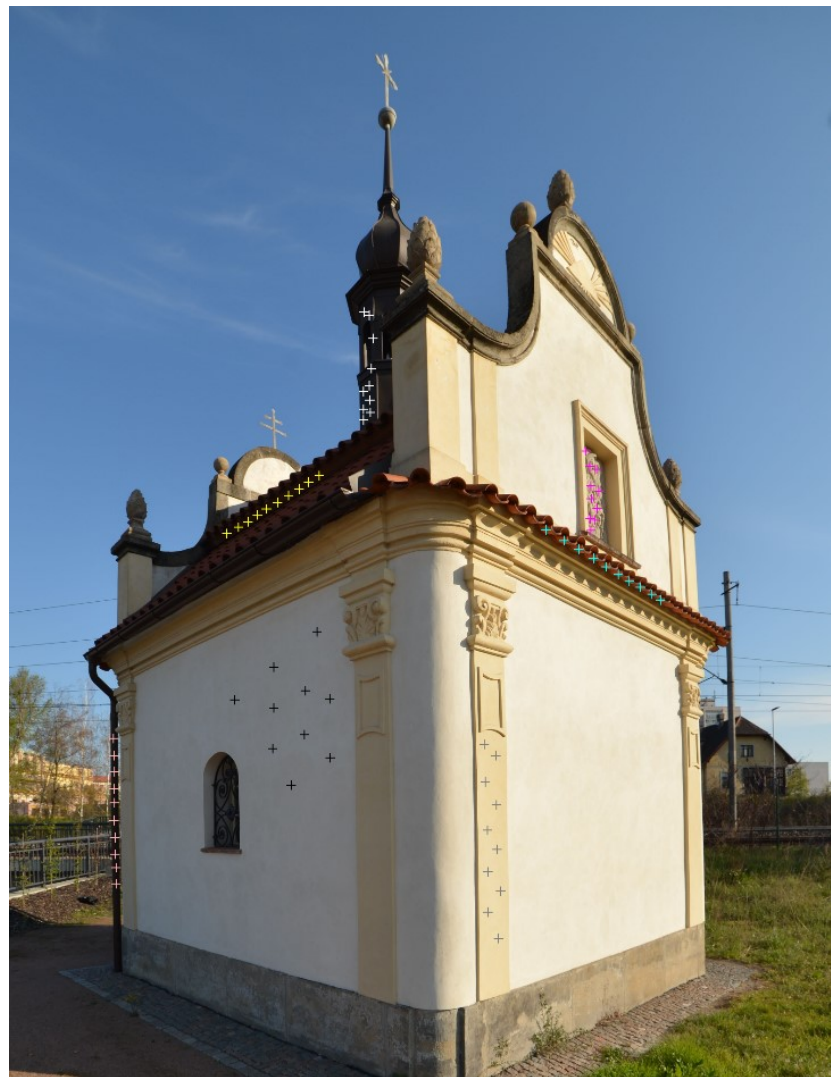

b) $2^{\text {nd }}$ and $3^{\text {rd }}$ sides (scan 2)

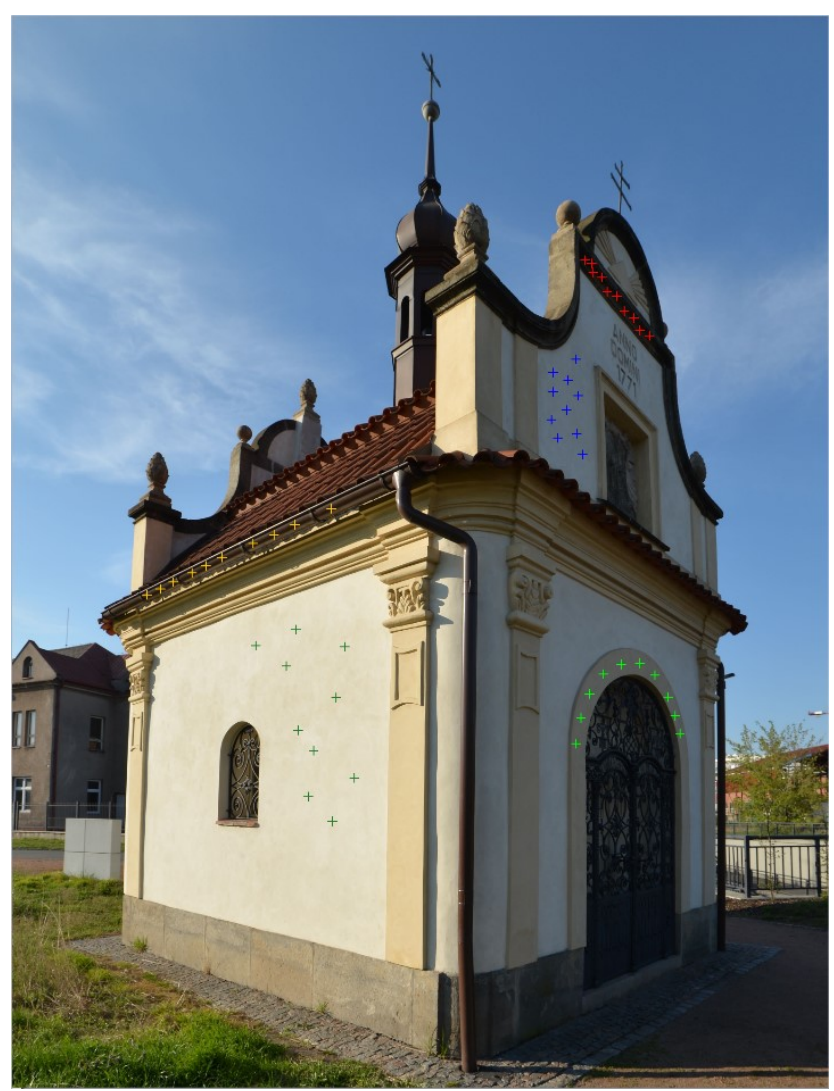

d) $4^{\text {th }}$ and $1^{\text {st }}$ sides $(\operatorname{scan} 4)$

Figure 2: Scans of the chapel 
3-dimensional vector $\boldsymbol{a}_{1}^{I}$ is the vector of true coordinates $n_{i}$ points on $\mathrm{i}$-th side of object in a coordinate system of $\mathrm{i}$-th device position. Analogous $\boldsymbol{a}_{1}^{I}$ is $3 n_{i+1}$-dimensional vector of $n_{i+1}$ points on $(i+1)$-th side of an object in a coordinate system of $i$-th device position.

From layout of measurement we can obtain constraint function

$$
\boldsymbol{g}=\left[\begin{array}{l}
\boldsymbol{g}_{2}^{I I}\left(\gamma_{2}, \boldsymbol{T}_{2}\right) \\
\boldsymbol{g}_{3}^{I I}\left(\gamma_{2}, \boldsymbol{T}_{2}\right) \\
\boldsymbol{g}_{3}^{I I}\left(\gamma_{3}, \boldsymbol{T}_{3}\right) \\
\boldsymbol{g}_{4}^{I I I}\left(\gamma_{3}, \boldsymbol{T}_{3}\right) \\
\boldsymbol{g}_{4}^{I V}\left(\gamma_{4}, \boldsymbol{T}_{3}\right) \\
\boldsymbol{g}_{1}^{I V}\left(\gamma_{4}, \boldsymbol{T}_{3}\right)
\end{array}\right]=\left[\begin{array}{l}
\boldsymbol{a}_{2}^{I I}-\gamma_{2}-\boldsymbol{T}_{2} \boldsymbol{a}_{2}^{I} \\
\boldsymbol{a}_{3}^{I I}-\gamma_{2}-\boldsymbol{T}_{2} \boldsymbol{a}_{3}^{I} \\
\boldsymbol{a}_{3}^{I I}-\gamma_{3}-\boldsymbol{T}_{3} \boldsymbol{a}_{3}^{I} \\
\boldsymbol{a}_{4}^{I I I}-\gamma_{3}-\boldsymbol{T}_{3} \boldsymbol{a}_{4}^{I} \\
\boldsymbol{a}_{4}^{I V}-\gamma_{4}-\boldsymbol{T}_{4} \boldsymbol{a}_{4}^{I} \\
\boldsymbol{a}_{1}^{I V}-\gamma_{4}-\boldsymbol{T}_{4} \boldsymbol{a}_{1}^{I}
\end{array}\right]=0
$$

Let the true model of our chapel in coordinate system $\mathrm{S}_{0}$ be given. We will consider that the base of our chapel is is an equilateral trapezoid with length of sides $4.500 \mathrm{~m}$, $4.300 \mathrm{~m}$ and $5.051 \mathrm{~m}$. Now we will set origins of coordinate systems $\mathrm{S}_{2}, \mathrm{~S}_{3}$, and $\mathrm{S}_{4}$, see Tab. 1 .

Further, we consider that matrices $T_{2}, T_{3}, T_{4}$ are given as

$\boldsymbol{T}_{i}=\left(\begin{array}{cc}\boldsymbol{R}_{i} & 0 \\ 0 & 1\end{array}\right), \quad \boldsymbol{R}_{i}=\left(\begin{array}{cc}c_{i} & s_{i} \\ -s_{i} & c_{i}\end{array}\right)$

where $c_{i}=\cos \left(\theta_{i}\right), \quad s_{i}=\sin \left(\theta_{i}\right)$ e.g. transformation do not change vertical position of chapel.

According to our experiment and obvious uncertainty of $3 \mathrm{D}$ camera, we consider that the standard deviation $\sigma_{d}=2 \mathrm{~cm}$. Of course such value is large measurement error.

A following numerical study will be made. Firstly we transform coordinates $\boldsymbol{a}_{0}$ of points on true trapezoid model from coordinate system $\mathrm{S}_{0}$ to $\mathrm{S}_{1}$.

We will use transformation: $\boldsymbol{a}_{1}=\boldsymbol{\gamma}_{1}+\boldsymbol{T}_{1} \boldsymbol{a}_{0}$.

We set $\quad \gamma_{1}=[44.000,90.000]^{\prime}$ and $\theta_{i}=\frac{4}{3} \pi \Rightarrow$ $\boldsymbol{R}_{1}=\left(\begin{array}{c}\cos \left(240^{\circ}\right), \sin \left(240^{\circ}\right) \\ -\sin \left(-240^{\circ}\right), \cos \left(240^{\circ}\right)\end{array}\right)$.

Using formulas $a_{2}=\gamma_{2}+\boldsymbol{T}_{2} \boldsymbol{a}_{1}, \boldsymbol{a}_{3}=\boldsymbol{\gamma}_{3}+\boldsymbol{T}_{3} \boldsymbol{a}_{2}$, $\boldsymbol{a}_{4}=\boldsymbol{\gamma}_{4}+\boldsymbol{T}_{4} \boldsymbol{a}_{3}$ we obtained coordintes of points in every coordinate system $\mathrm{S}_{1}, \mathrm{~S}_{2}, \mathrm{~S}_{3}, \mathrm{~S}_{4}$. From data $\boldsymbol{a}_{1}, \boldsymbol{a}_{2}$, $\boldsymbol{a}_{3}, \boldsymbol{a}_{4}$ it is possible to obtained only points $\boldsymbol{a}_{1}^{I}, \boldsymbol{a}_{2}^{I}, \boldsymbol{a}_{3}^{I}$, $\boldsymbol{a}_{4}^{I}$ that lie only on first, second, third or fourth side of our object.

To these exact coordinates we add measurement errors by generating independent epsilon errors. With respect to the origins of coordinate systems we then extracted the simulated (measured) values of $\mathbf{Y}$, cf. model (1).

The simulated values are available on the website (Nedvědová 2019).

Table 1 presents the transformation parameters between start and target coordinate systems.

Part of the coordinates of identical points are given in Table 3.
Table 1: True transformation parameters

\begin{tabular}{|c|c|c|c|}
\hline \multicolumn{4}{|c|}{ Sides } \\
\hline Scans & 1,2 to 2,3 & 2,3 to 3,4 & 4,1 to 1,2 \\
\hline Shift & $-50,30$ & 65,125 & 118,38 \\
\hline$\theta_{i}$ & $65^{\circ}$ & $148^{\circ}$ & $244^{\circ}$ \\
\hline $\begin{array}{c}\text { Rota- } \\
\text { tion }\end{array}$ & $\begin{array}{cc}0.42 & 0.91 \\
-0.91 & 0.42 \\
\end{array}$ & $\begin{array}{cc}-0.85 & 0.53 \\
-0.53 & -0.85 \\
\end{array}$ & $\begin{array}{cc}-0.44 & -0.90 \\
0.90 & -0.44\end{array}$ \\
\hline
\end{tabular}

\subsection{The second step: HSV simulation}

First, we select points of the same type that appear in photos taken from different locations.

For 12 color groups with ten-point color, we obtained HSV measurements in two scans for every chaple's side. For example, the fifth color group was created from points on the stone plinth of the chapel. Points were focused in the 1 st and 2 nd scans.

The diagram in the figure 3 shows information about in which scans the color groups were selected and targeted. By analyzing data containing 12 times 10 points, we estimate the variability of HSV components. Averages and standard deviations of HSV measurement for all color group are given in Tab. 2.

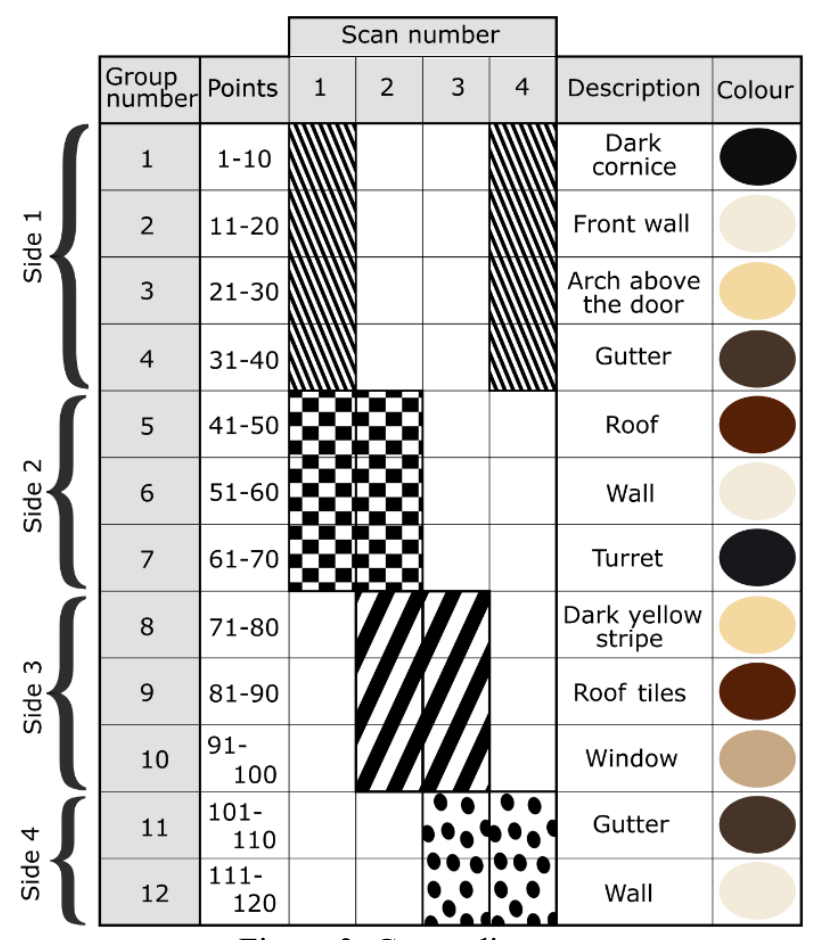

Figure 3: Group diagram 
Table 2: Pairs of HSV measurements

\begin{tabular}{|c|c|c|c|}
\hline & \multicolumn{3}{|c|}{ Average HSV } \\
\hline & $\mathrm{H}$ & $\mathrm{S}$ & $\mathrm{V}$ \\
\hline Group 5, Scan 1 & 49.2156 & 26.7981 & 3.3147 \\
\hline Group 5, Scan 2 & 73.9066 & 26.1388 & 3.3262 \\
\hline Group 6, Scan 1 & 48.3860 & 25.6160 & 5.2158 \\
\hline Group 6 , Scan 2 & 72.6436 & 26.8238 & 5.2061 \\
\hline & \multicolumn{3}{|c|}{ Standard deviation } \\
\hline & $\mathrm{H}$ & $\mathrm{S}$ & $\mathrm{V}$ \\
\hline Group 5, Scan 1 & 0.3631 & 0.5231 & 0.1314 \\
\hline Group 5, Scan 2 & 0.5552 & 0.2982 & 0.1328 \\
\hline Group 6 , Scan 1 & 0.2376 & 0.3285 & 0.7499 \\
\hline Group 6, Scan 2 & 0.3480 & 0.1971 & 0.7570 \\
\hline
\end{tabular}

We created a matrix of differences in HSV values in these two scans, which has a dimension of $120 \times 3$. For these measurements, we have obtained a $3 \times 3$ covariance matrix that describes the variability and dependence of HSV components. This matrix is shown in formula (6).

$V(H, S, V)=\left(\begin{array}{ccc}0.14 & 0.0004 & 0.003 \\ 0.0004 & 0.0142 & 0.0014 \\ 0.003 & 0.0014 & 0.0016\end{array}\right)$

However, we did not use this matrix for simulation. For all 12 color groups, we determined the variance matrices using 10 points measured in two scans:

$$
\begin{aligned}
& V_{1}(H, S, V)=\left(\begin{array}{lll}
0.0665 & 0.0028 & 0.0040 \\
0.0028 & 0.0199 & 0.0020 \\
0.0040 & 0.0020 & 0.0005
\end{array}\right) \\
& V_{2}(H, S, V)=\left(\begin{array}{lll}
0.2086 & 0.0943 & 0.0587 \\
0.0943 & 0.4476 & 0.1554 \\
0.0587 & 0.1554 & 0.0959
\end{array}\right) \cdot 10^{-3} \\
& V_{3}(H, S, V)=\left(\begin{array}{ccc}
0.0055 & -0.0001 & -0.0002 \\
-0.0001 & 0.0007 & -0.0003 \\
-0.0002 & -0.0003 & 0.0002
\end{array}\right) \\
& V_{4}(H, S, V)=\left(\begin{array}{ccc}
0.5728 & -0.0153 & 0.0033 \\
-0.0153 & 0.0052 & 0.0004 \\
0.0033 & 0.0004 & 0.0002
\end{array}\right) \\
& V_{5}(H, S, V)=\left(\begin{array}{lll}
0.0003 & 0.0007 & 0.0000 \\
0.0007 & 0.0059 & 0.0008 \\
0.0000 & 0.0008 & 0.0003
\end{array}\right) \\
& V_{6}(H, S, V)=\left(\begin{array}{ccc}
0.0027 & -0.0011 & -0.0004 \\
-0.0011 & 0.0006 & 0.0003 \\
-0.0004 & 0.0003 & 0.0002
\end{array}\right) \\
& V_{7}(H, S, V)=\left(\begin{array}{ccc}
0.0005 & -0.0004 & -0.0000 \\
-0.0004 & 0.0034 & 0.0003 \\
-0.0000 & 0.0003 & 0.0001
\end{array}\right) \\
& V_{8}(H, S, V)=\left(\begin{array}{ccc}
0.0034 & 0.0111 & -0.0088 \\
0.0111 & 0.2452 & -0.0339 \\
-0.0088 & -0.0339 & 0.0446
\end{array}\right) \cdot 10^{-3}
\end{aligned}
$$

$$
\begin{aligned}
& V_{9}(H, S, V)=\left(\begin{array}{ccc}
0.0062 & -0.0212 & -0.0022 \\
-0.0212 & 0.5339 & -0.0983 \\
-0.0022 & -0.0983 & 0.4110
\end{array}\right) \cdot 10^{-3} \\
& V_{10}(H, S, V)=\left(\begin{array}{ccc}
0.0001 & -0.0002 & -0.0000 \\
-0.0002 & 0.0010 & -0.0006 \\
-0.0000 & -0.0006 & 0.0012
\end{array}\right) \\
& V_{11}(H, S, V)=\left(\begin{array}{ccc}
0.0002 & 0.0001 & -0.0000 \\
0.0001 & 0.0060 & -0.0017 \\
-0.0000 & -0.0017 & 0.0009
\end{array}\right) \\
& V_{12}(H, S, V)=\left(\begin{array}{ccc}
0.0513 & -0.0375 & 0.0122 \\
-0.0375 & 0.2651 & -0.1361 \\
0.0122 & -0.1361 & 0.1053
\end{array}\right) \cdot 10^{-3}
\end{aligned}
$$

We can proceed as follows.

To the points simulated by transformation parameters given in Table 1, HSV values simulation was added. We selected the exact HSV value for any point on our object. We randomly selected one of the 12 covariance matrices $\mathrm{V}_{1}$ to $\mathrm{V}_{12}$. Using this randomly chosen covariance matrix, we simulated measurements for two different scans twice. During the simulation we assumed normal error distribution of HSV and chosen covariance matrix V. We used simple simulation technique for normal data with estimated prespecified covariance matrix. For detail see (Kaiser, 1962).

We use funcction $\mathrm{R}=\operatorname{mvnrnd}(\mu, \Sigma)$, that returns an $\mathrm{N}$ by-D matrix $\boldsymbol{R}$ of random vectors chosen from the multivariate normal distribution with mean vector $\boldsymbol{\mu}$, and covariance matrix $\Sigma$.

\section{NUMERICAL STUDIES}

\subsection{Estimation in our test problem}

The ICP method is applied to our data set. The estimated parameters are presented on the website (Nedvědová 2019).

According to the articles (Amiri-Simkooei 2018) and (Marek 2015) we calculate the transformation parameters for the task. We applied the ICP method from Point Cloud Library (Rusu and Cousins 2011) to find pairs of identical points between scans based on the similarity of HSV values to estimate the transformation parameters.

We just decide to use the HSV color model on base of our previous research (Chmelar and Benkrid 2014) and (Chmelar, Beran and Kudriavtseva 2015), where for a color detection form static frames the HSV model overcomes standard used color models. Its advantage lies in color description by only one channel. Other channels describes a concrete color's properties.

The following figure shows comparison between RGB Fig. 4 (a) and HSV Fig. 4 (b) color space for the exact color. When we match similar color from different chapel's sides the bigger color span in the color space it is more suitable, but when the ICP algorithm's parameters are properly set, than the precise match is achieved. 
Table 3: Simulation of HSV: identical points

\begin{tabular}{|l|c|c|c|}
\hline & \multicolumn{3}{|c|}{ Point: X, Y, Z } \\
& & H, V \\
\hline No 51: & 60.6841 & 7.1315 & 47.2399 \\
Scan 1 & 48.7407 & 26.1132 & 4.2658 \\
\hline No 51: & 10.8373 & 27.6623 & 84.1735 \\
Scan 2 & 73.4339 & 26.4070 & 2.6310 \\
\hline No 52: & 68.2305 & 3.1235 & 46.3298 \\
Scan 1 & 48.4720 & 25.7026 & 4.5565 \\
\hline No 52: & 11.1249 & 27.2490 & 86.7095 \\
Scan 2 & 73.4181 & 26.4010 & 2.7851 \\
\hline No 53: & 59.4553 & 5.6575 & 47.4210 \\
Scan 1 & 48.1522 & 25.3072 & 4.2163 \\
\hline No 53: & 10.3987 & 31.7112 & 86.7758 \\
Scan 2 & 73.1719 & 26.5519 & 2.8363 \\
\hline No 54: & 63.3525 & 4.0797 & 47.2996 \\
Scan 1 & 48.0840 & 25.1802 & 4.9363 \\
\hline No 54: & 9.0201 & 35.0025 & 85.3573 \\
Scan 2 & 73.4261 & 26.3800 & 3.1580 \\
\hline No 55: & 65.6075 & 3.1142 & 46.2436 \\
Scan 1 & 48.6650 & 25.9867 & 5.0952 \\
\hline No 55: & 11.7636 & 23.8849 & 88.5562 \\
Scan 2 & 73.2439 & 26.5146 & 3.1231 \\
\hline No 56: & 56.8741 & 5.8002 & 47.3855 \\
Scan 1 & 48.3353 & 25.5887 & 5.1940 \\
\hline No 56: & 10.1819 & 26.9011 & 88.6639 \\
Scan 2 & 73.1590 & 26.5272 & 3.2359 \\
\hline No 57: & 54.3685 & 11.6568 & 51.7027 \\
Scan 1 & 48.6171 & 25.9424 & 5.6725 \\
\hline No 57: & 10.9817 & 28.4105 & 83.5378 \\
Scan 2 & 73.3495 & 26.4477 & 3.2783 \\
\hline No 58: & 66.4225 & 0.5393 & 44.5333 \\
Scan 1 & 48.1491 & 25.3256 & 5.7490 \\
\hline No 58: & 11.7224 & 26.7111 & 88.0017 \\
Scan 2 & 73.4699 & 26.3413 & 2.9403 \\
\hline No 59: & 64.3876 & 4.5814 & 46.5366 \\
Scan 1 & 48.4461 & 25.7048 & 6.0703 \\
\hline No 59: & 11.3594 & 27.9017 & 87.5473 \\
Scan 2 & 73.2436 & 26.4897 & 2.7728 \\
\hline No 60: & 59.5184 & 6.1586 & 46.3937 \\
Scan 1 & 48.1983 & 25.3090 & 6.4026 \\
\hline No 60: & 10.0160 & 27.1193 & 89.3212 \\
Scan 2 & 73.1467 & 26.5443 & 2.6745 \\
\hline
\end{tabular}
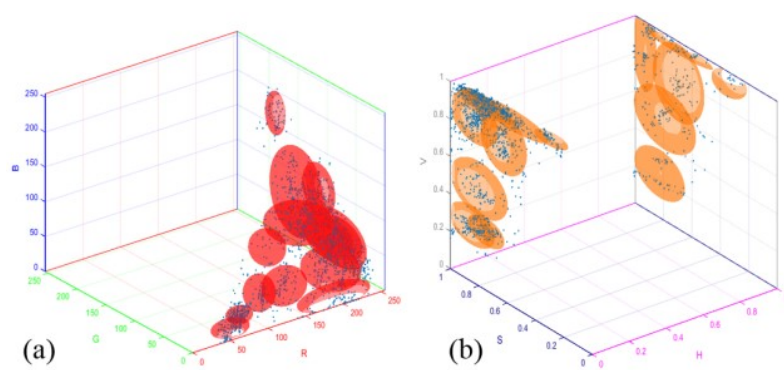

Figure 4: Color space span for exact color, (a) RGB model, (b) HSV model
The testing dataset includes four registration cases. Each case describes registration of two chapel's sides with identical points, sides $1-2,2-3,3-4$ and $4-1$.

\section{CONCLUSION}

In this paper, we presented the process of simulation of data for a registration problem. We designed the testing example for multi-stage $3 \mathrm{D}$ coordinate transformations.

\section{ACKNOWLEDGMENTS}

The paper was supported by grant SGS 2019_021 of the University of Pardubice.

\section{REFERENCES}

Amiri-Simkooei, A. R., 2018. Parameter estimation in 3D affine and similarity transformation: implementation of variance component estimation. Journal of Geodesy, 92 (11), 1285-1297.

Bataineh, S., Bahillo, A., Díez, L., Onieva, E., and Bataineh, I., 2016. Conditional Random FieldBased Offline Map Matching for Indoor Environments. Sensors, 16(8).

Burguera, A., Gonzalez, Y., and Oliver, G., 2008. The likelihood field approach to sonar scan matching. IEEE/RSJ International Conference on Intelligent Robots and Systems, pp. 2977-2982.

He, Y., Liang, B., Yang, J., Li, S., and He, J. 2017. An Iterative Closest Points Algorithm for Registration of 3D Laser Scanner Point Clouds with Geometric Features. Sensors, 17(8).

Chmelar, P., and Benkrid, A., 2014. Efficiency of HSV over RGB Gaussian Mixture Model for fire detection. 24th International Conference Radioelektronika, pp. 1-4

Chmelar, P., Beran, L. and Kudriavtseva, N. 2015. The laser color detection for 3D range scanning using Gaussian mixture model. 25th International Conference Radioelektronika pp. 248-253

Lu, F., and Milios E., 1997. Robot pose estimation in unknown environments by matching $2 \mathrm{D}$ range scans. Journal of Intelligent and Robotic Systems [online]. 18(3), 249-275.

Kaiser, H. F., \& Dickman, K., 1962. Sample and Population Score Matrices and Sample Correlation Matrices from an Arbitrary Population Correlation Matrix, Psychometrika, 27 (2), 179-182.

Magnusson, M., 2013. The three-dimensional normaldistributions transform: an efficient representation for registration, surface analysis, and loop detection. Dissertation thesis. Örebro university.

Marek J., Rak J., and Jetensky P., 2015. Statistical solution of $3 \mathrm{D}$ transformation problem. In: V. Skala, ed. Proceedings of 23rd International Conference in Central Europe on Computer Graphics, Visualization and Computer Vision, WSCG 2015, pp. 85-90. Jun 8-13, Pilsner (Czech republic).

Mitra, N. J., Gelfand, N., Pottmann, H., and Guibas, L., 2004. Registration of point cloud data from a geometric optimization perspective. 
Eurographics/ACM SIGGRAPH symposium on Geometry processing - SGP 04, p. 22.

Montesano, L., Minguez, J., and Montano, L., 2005. Probabilistic scan matching for motion estimation in unstructured environments. IEEE/RSJ International Conference on Intelligent Robots and Systems, pp. 3499-3504

Nedvědová, M., 2019. Estimates of unknown transformation parameters in terrestrial measurements: One simulated problem. Available from: http://hsv-transform.estetickecharakteristiky.eu [Accessed 10 July 2019]

Nüchter, A., 2009. 3D robotic mapping: the simultaneous localization and mapping problem with six degrees of freedom. Heidelberg: Springer.

Olsen, M. J., Johnstone, E., Kuester, F., Driscoll, N., and Ashford, S. A., 2011. New Automated Point-Cloud Alignment for Ground-Based Light Detection and Ranging Data of Long Coastal Sections. Journal Of Surveying Engineering, 137(1), 14-25.

Rusu, R., and Cousins, S., 2011. 3D is here: Point Cloud Library (PCL). IEEE International Conference on Robotics and Automation, pp. 1-4 